\title{
Stellungnahme der DGP zur Pressemitteilung des National Heart, Lung, and Blood Institute zur PANTHER-Studie bei IPF-Patienten vom 21.10.2011
}

\section{Comments of the DGP on the Press Release dated 21.10.2011 of the National Heart, Lung and Blood Institute about the PANTHER Study on IPF Patients}

Autoren

Institute
J. Behr ${ }^{1}$, U. Costabel ${ }^{2}$, H. Worth ${ }^{3}$

${ }^{1}$ Abteilung für Pneumologie, Allergologie, Schlaf- und Beatmungsmedizin, Berufsgenossenschaftliches Universitätsklinikum Bergmannsheil GmbH, Bochum

${ }^{2}$ Abteilung Pneumologie/Allergologie, Ruhrlandklinik Essen

${ }^{3}$ Medizinische Klinik 1, Klinikum Fürth,

für den Vorstand der DGP
Bibliografie

DOI http://dx.doi.org/

10.1055/s-0031-1291535

Pneumologie 2011; 65: 724-725

(c) Georg Thieme Verlag KG

Stuttgart · New York

ISSN 0934-8387

Korrespondenzadresse

Prof. Dr. med Jürgen Behr

Abteilung für Pneumologie, Allergologie, Schlaf- und Beatmungsmedizin Berufsgenossenschaftliches Universitätsklinikum

Bergmannsheil GmbH

Bürkle-de-la-Camp-Platz 1

44789 Bochum

Juergen.behr@bergmannsheil.de
Die PANTHER-Studie ist eine amerikanische Studie, die randomisiert, kontrolliert, prospektiv in drei Behandlungsarmen Therapieoptionen der idiopathischen pulmonalen Fibrose (IPF) untersucht:

1. Prednison plus Azathioprin plus N-Acetylcystein (sog. „Triple-Therapie“),

2. N-Acetylcystein-Monotherapie,

3. Placebo. Am 21 Oktober 2011 gab das National Heart, Lung, and Blood Institute (NHLBI) bekannt, dass der „Triple-Therapie“-Arm der PANTHER-Studie wegen Sicherheitsbedenken geschlossen wurde. Im Rahmen einer Zwischenanalyse nach Einschluss von 238 der geplanten 390 IPF-Patienten zeigten sich im Triple-Therapie-Arm im Vergleich zu Placebo eine erhöhte Mortalität (11\% versus 1\%), vermehrt Hospitalisationen (29\% versus $8 \%$ ) und gehäuft schwerwiegende Nebenwirkungen (31\% versus 9\%). Die Studie wird nun mit den beiden verbleibenden Behandlungsarmen $\mathrm{N}$-Acetylcystein-Monotherapie und Placebo fortgesetzt.

Auch wenn zum jetzigen Zeitpunkt noch keine abschließende Bewertung der Beobachtung möglich ist, sind diese Daten beunruhigend. Allerdings ist bisher unklar, wie die außergewöhnlich niedrige Mortalität von $1 \%$ in der Placebogruppe zu erklären ist, ob die demografischen Ausgangsdaten mit früheren IPF-Studien vergleichbar sind, welche Todesursachen aufgetreten sind und ob Infektionen oder akute Exazerbationen vermehrt bei älteren Patienten unter Immunsuppression aufgetreten sind. Auf der Basis einer Pressemitteilung können daher keine Therapieempfehlungen abgegeben werden. Dennoch halten wir die Befunde für so bedeutsam, dass bis zum Vorliegen klärender Analysen der Studiendaten folgende Vorgehensweisen für die Behandlung von IPF-Patienten erwogen werden müssen:
1. Auch wenn bisher unklar ist, ob akute Exazerbationen infektionsbedingt die im Vergleich zu Placebo erhöhte Mortalität verursachten, kann die bekannte immunsuppressiven Wirkungsweise von Azathioprin und Prednisolon eine plausible Erklärung darstellen. Patienten mit neu diagnostizierter IPF sollten daher über Behandlungsmöglichkeiten unter Bezugnahme auf die PANTHER-Presseerklärung umfassend aufgeklärt werden. Es ist zu erwarten, dass Patienten sich angesichts der Datenlage gegen die Einleitung der Triple-Therapie entscheiden werden.

2. Bei Patienten mit IPF, die bereits mit einer Kombination aus Prednisolon, Azathioprin behandelt werden, sollte ein Absetzen der Therapie unter Berücksichtigung von Verträglichkeit, Nebenwirkungen, Komplikationen und des klinischen Verlaufs erwogen und mit dem Patienten besprochen werden. Hierbei und in jedem Einzelfall sind die Zuverlässigkeit der IPF-Diagnose, das Ansprechen der Therapie des Patienten und das Risiko einer Krankheitsprogression nach Absetzen der Therapie zu beachten. Argumente, die für das Absetzen der Immunsuppression sprechen, sind:

a) Progression der Erkrankung trotz der Therapie

b) therapieassoziierte Komplikationen, insbesondere Infektionen

c) Therapiedauer unter 6 Monate

3. Für IPF-Patienten mit über 6 Monate bestehender Krankheitsstabilität unter Prednisolon und Azathioprin muss in jedem Einzelfall eine Nutzen-Risiko-Abwägung bezüglich eines Therapieabbruchs erfolgen.

4. Für die Gabe von N-Acetylcystein ergeben sich aus den aktuellen Daten keine Änderungen hinsichtlich des Einsatzes bei IPF-Patienten. 
5. Für Patienten mit anderen interstitiellen Lungenerkrankungen als einer IPF ergeben sich aus den aktuellen Daten ebenfalls keine Änderungen, wenngleich eine erhöhte Aufmerksamkeit bezüglich möglicher Nebenwirkungen angeraten ist. Auch für Patienten mit nicht-spezifischer interstitieller Pneumonie und exogen allergischer Alveolitis muss vor dem Hintergrund der NHLBI-Daten eine individuelle Nutzen-Risiko-Abwägung über die Fortsetzung oder den Abbruch der Behandlung mit Azathioprin und Prednisolon entscheiden. 